\title{
Inhaltsverzeichnis
}

\section{Versicherungs-Statistik für 1907}

Einleitung .. . . . . . . . . . . . . . . .

Allgemeiner Überblick. . . . . . . . . . . . . . .

Gruppe I: Lebens- und Krankenversicherung . . . 18

Gruppe II: Unfall- und Haftpflichtversicherung . . $36^{*}$ Gruppe III : Vieh- und Hagelversicherung . . . . 42*

Gruppe IV: Feuerversicherung sowie Versicherung gegen Sturmschäden, Wasserleitungsschäden und Einbruchdiebstabl . . . . $52^{*}$

Gruppe V: Glasversicherung, Kautions- und Garantieversicherung, Kredit-, Maschinen-, Kasko-

Tafel. versicherung, Kredit-, Maschinen-, Kasko-
versicherung usw. . . . . . . . . . $62^{*}$

\section{Tabel1en}

\section{Gruppe I. Lebense und Kranken- versicherung}

Verzeichnis der Abkürzungen für die Namen der Unternehmungen . . . . . . . . . . .

\section{A. Versicherungsbestand Ende 1907 Deutsche allgemeine Unternehmungen}

$$
\text { Grobe Versicherung }
$$

Tab. I 1: Die überbaupt tubernommenen Versicherungen nach Versicherungsarten . . . . . . .

n I 2: Die in Ruckdeckung gegebenen Versicherungen und die Versicherungen fär eigene Rechnung nach Versicherungsarten ... .

" I 3: Bestand der selbst abgeschlossenen und der in Rückdeckung übernommenen Versicherungen auf den Todesfall, Durchschnittsbeträge und Gewinnbeteiligung . . . . .

" I 4: Bestand der selbst abgeschlossonen und der in Ruckdeckung übernommenen Versicherungen suf den Lebensfall, Durchschnittsbeträge und Gewinnbeteiligung . . . . .

"I 5: Bestand der selbst abgeschlossenen und der in Ruckdeckung ubernommenen Rentenversicherungen, Durchschnittsbeträge und Gewinnbeteiligung. . . . . . . . . .

" I6: Bestand der selbst abgeschlossenen und der in Rückdeckung übernommenen Invaliditätsversicherangen, Durchschnittsbeträge und Gewinnbeteiligung. . . . . . . . . .

Tab.I 7: Bestand der selbst abgeschlossenen und der in Rückdeckung übernommenen Krankenver-
Seite $7^{*}$ $9^{*}$ $8^{*}$ $52^{*}$ sicherungen, Durchschnittsbeträge und Gewinnbeteiligung. . . . . . . . . 21

\section{Kleine Versicherung}

I 8: Bestand der überhaupt übernommenen, der in Räckdeckung gegebenen Versicherungen und der Versicherungen für eigene Rechnung

, I9: Bestand der selbst abgeschlossenen und der in Rückdeckung übernommenen Versicherungen auf den Todesfall, Durchschnittsbeträge and Gewinnbeteiligung . . . . .

I 10: Bestand der selbst abgeschlossenen und der in Rückdeckung ubernommenen Versicherungen anf den Lebensfall, Durchschnittsbeträge und Gewinnbeteiligung . . . . . 25

\section{Versicherungseinrichtungen größerer deutseher Berufsvereinigungen}

"I11: Bestand der Versicherungen, Darchschnittsbeträge und Gewinnbeteiligung . . . . . 26

Grobe Versicherung - Deatscher Bestand

Tab.I 12: Die uberhaupt übernommenen Versicherungen nach Versicherungsarten - Verhăltnis des dentschen Geschäfts zum ganzen Geschäfte. . . . . . . . . . . . .

" 113: Die in Rückdeckung gegebenen Versicherungen und die Versicherungen fur eigene Rechnung nach Versicherungsarten. . . .

" I14: Bestand der selbst abgeschlossenen und der in Rückdeckung übernommenen Versicherungen auf den Todesfall, Durchschnittsbetrăge und Gewinnbeteiligung . . . . .

"I 15: Bestand der Versicherangen aufden Lebensfall, Durchschnittsbeträge und Gewinnbeteiligung

"I16: Bestand der selbst abgeschlossenen und der in Rückdeckung übernommenen Rentenversicherungen, Durchschnittsbeträge und Gewinnbeteiligung - Invaliditätsversicherung

Kleine Versicherung - Deutscher Bestand "1 17: Bestand der tuberhaupt übernommenen, der in Räckdeckung gegebenon Versicherungen und der Versicherungen für eigene Rechnung - Verhältnis des deutschen Geschäfts zum ganzen Geschäfte . . . . . . . . . .

" 1 18: Bestand der Versicherungen, Durchschnittsbeträge und Gewinnbeteiligung . . . . . 35 
B. Bewegung im Versicherungsbestand im Jahre 1907

Dentsche allgemeine Unternehmungen Grobe Versicherung

Tab. I19: Bewegung in der Versicherung anf den Todesfall . . . . . . . . . . . . . " I 20: Bewegung in der Versicherung anf den Lebensfall . . . . . . . . . . . 44

" I 21: Bewegung in der Rentenversicherung. . . 52

$$
\text { Kloine Versicherung }
$$

"122: Bewegung in der Versicherung anf den Todesfall . . . . . . . . . . . . .

"I 23: Bewegung in der Versicherung auf den Lebensfall . . . . . . . . . . .

Versicherungseinrichtungen größerer deutscher Berufsvereinigungen

"I 24: Bewegung in der Versicherung . . . . .

\section{Ausländische Gesellschaften}

GroBe Versicherung - Deutscher Bestand

"125: Bewegung in der Versicherang anf den Todesfall . . . . . . . . . . . . .

"I 26: Bewegung in der Versicherang anf den Lebensfall . . . . . . . . . . . .

"I 27: Bewegung in dor Rentenversicherang . .

Kleine Versicherung - Dentscher Bestand

"128: Bewegung in der Versicherung auf den Todesfall und anf den Lebensfall

\section{Betriebsrechnung furr 1907}

Deutsche allgemeine Unternehmungen

\section{Grobe Versicherang}

n I 29: Gewinn- und Verlustrechnung . . . . .

" 130 : Die Einnahmen aus Prämien und die Vergütung der Rückversicherer sowie die Ausgaben für Versicherungsfälle des Geschäftsjahrs und für Rückversicherung . . . . .

n I 31: Das finanzielle Ergebnis aus der Sterblichkeit

Kleine Versicherung

n I 32: Gewinn- und Verlustrechnung . . . . .

"I 33: Die Einnahmen aus Prämien und die Vergütung der Ruckversicherer sowie die Ausgaben für Versicherungsfälle des Geschäftsjahrs und für Rückversicherung. . . . 102

\section{Versicherungseinrichtungen größerer deutscher Berufsvereinigungen}

"I 34: Gewinn- und Verlustrechnung . . 106

"135: Die Einnahmen aus Prämien und die Vergütung der Rückversicherer sowie die Ausgaben für Versicherungsfälle des Geschäftsjahrs und für Rückversicherung . . . . 11

\section{Auslandische Gesellschaften}

Grobe und kleine Versicherung

Tab.I 36: Gewinn- and Verlustrechnung für das Gesamtgeschäft . . . . . . . . . . .

"I 37: Deutsches Geschäft, die Einnahmen ans Prämien and die Vergütung der Rückversicherer sowie die Ausgaben für Versicherungsfalle des Geschäftsjahrs und für Rückversicherung. . . . . . . . . . . . 117

D. Bilanz fur den SchluB des Jahres 1907 Deutsche allgemeine Unternehmangen

" 1 38: Bilanz . . . . . . . . . . . . . . . 120

" 139: Die Art der Kapitalanlagen und der Aktivforderungen . . . . . . . . . . . . 128

"I 40: Die Prämienreserven und die Prämienäberträge nach Versicherungsarten . . . 134

" I 41 : Die Verteilung des Gewinns . . . . . . 138

n I 42: Die Art und der Betrag der Ende 1907 vorhandenen Wertpapiere (Nennwert) . . . 142

Versicherungseinrichtnngen größerer deutscher Berufsvereinigungen

"I 13: Bilanz . . . . . . . . . . . . . . 146

"144: Die Art der Kapitalanlagen und der Aktivforderungen . . . . . . . . . . . . 150

" 145: Die Verteilung des Gewinns . . . . . . 152

" 146 : Die Art und der Betrag der Ende 1907 vorhandenen Wertpapiere (Nennwert). . . 154

Auslandische Gesellschaften

" 147: Bilanz . . . . . . . . . . . . . 156

" 148: Die Art der Kapitalanlagen und der Aktivforderungen . . . . . . . . 160

\section{Gruppe II. Unfall- und Haftpflichț- versicherung}

Verzeichnis der Ablürzungen für die Namen der

Unternehmungen . . . . . . . . . . 163

Deutsche Unternehmungen

Tab. II 1: Die Zahl der Ende 1907 laufenden Versicherungen und die Prämien des Geschäftsjahrs 166

" II 2: Vergleich des Geschäftsjahrs mit dem Vorjahre . . . . . . . . . . . . . 165

" II 3: Die Betriebs-Einnahmen und Ausgaben und der Betriebsgewinn der Unfall- und Haftpflichtversicherung . . . . . . . . . 168

" II 4: Die Zahlungen für selbst abgeschlossene Versicherungen und die Vergütungen für in Rückdeckung übernommene Versicherungen 170

" II 5: Das Ergebnis der Vermögensverwaltung . 174

" II 6: Bilanz . . . . . . . . . . . . . 176

" II 7: Die Art der Kapitalanlagen and der Aktivforderungen . . . . . . . . . . . 178

n II 8: Die Verteilnng des Gewinns . . . . . 178

- II 9: Die Art und der Betrag der Ende 1907 vorhandenen Wertpapiere (Nennwert) . . . 180 


\section{Aus]ändisehe Gesellschaften}

\section{Deutsches Geschäft}

Tab. II 10: Die Zahl der Ende 1907 laufenden Versicherungen und die Prämien des Geschäftsjahrs . . . . . . . . . . . . .

"II11: Vergleich des Geschäftsjahṛ mit dem Vorjahre . . . . . . . .'. . . . .

"II12: Die Zahlungen für selbst abgeschlossene Versicherungen und die Vergütungen für in Rückdeckang übernommene Versicherungen

\section{Gessmtgesch $\bar{s} f t$}

" Il13: Die Betriebs-Einnahmen und Ausgaben der Unfall- und Haftpflichtversicherang . . . 184

"II 14: Bilanz . . . . . . . . . . . . . 186

" II 15: Die Art der Kapitalanlagen und der Aktivforderungen . . . . . . . . . 18

\section{Gruppe III. Landwirtschaftliche Vereicherung}

Verzeichnis der Abkürzangen für die Namen der Unternehmungen ...........

\section{Viehversicherung}

Tab. III 1: Umfang der Viehversicherung im Geschäftsjahre 1907 . . . . . . . . . . . . 191

n III 2: Zerlegung des Geschåfts nach Versicherungsarten . . . . . . . . . . 192

" III 3: Vergleich des Geschäftsjahrs mit dem Vorjahre. . . . . . . . . . . . . . 195

"III 4: Gewinn- und Verlustrechnung. . . . . 196

" III 5: Erläuterung der in die Gewinn- und Ver lustrechnung eingestellten Prämien und Schäden . . . . . . . . . . 200

" Ill 6: Bilanz . . . . . . . . . . . . . 202

" III 7: Die Art der Kapitalanlagen und der Aktivforderungen . . . . . . . . . . . 206

„III 8: Die Verteilung des Gewinns . . . . . . 210

" III 9: Die Art und der Betrag der Ende 1907 vorhandenen Wertpapiere (Nennwert) . . . 208

\section{Hagelversicherung}

" III 10: Umfang der Hagelversicherung im Geschäftsjahre 1907 . . . . . . . . . 211

" III 11: Bewegung in der Hagelversicherung . . 212

"III 12: Gewinn- und Verlustrechnung . . . . . 214

" III 13: Erlänterung der in die Gewinn- und Verlustrechnung eingestellten Prämien und Schăden . . . . . . . . . . . . 218

" III 14: Bilanz . . . . . . . . . . . . . 220

"III 15: Die Art der Kapitalanlagen und der Aktivforderungen . . . . . . . . . . . 224

" III 16: Die Verteilung des Gewinns . . . . . 228

"III 17: Die Art und der Betrag der Ende 1907 vorhandenen Wertpapiere (Nennwert) . . . 226
Gruppe IV. Feuerversicherung sowie die Versicherungen gegen Sturmschãden, Wasserleitungsechăden und Einbruchdiebstahl

Verzeichnis der Abkürzungen für die Namen der Unternehmungen . . . . . . . . . . . 229

\section{Versicherangsbestand Ende 1906 and Fnde 1907}

Dentsche Unternohmungen

Tab. IV 1: Zahl and Betrag der Feuerversicherungen - Gesamtgeschäft . . . . . . . 232 u. 236

, IV 2: Zahl und Betrag der Feuerversicherungen - Inländisches Geschäft . . . . . . 234

" IV 3: Zahl und Betrag der Sturmschäden- und der Wasserleitungsschädenversicherungen . . 237

" IV 4: Zahl und Betrag der Einbruchdiebstahlversicherungen - Gesamtgeschäft . 236 u. 240

IV 5: Zahl und Betrag der Einbruchdiebstahlversicherungen - Inländisches Geschäft . 242

\section{Ansländische Gesellschaften -} Deutsches Geschaft

„IV 6: Zahl und Betrag der Feuerversicherungon 244 "IV 7: Zahl und Betrag der Einbruchdiebstahlversicherungen . . . . . . . . . . 244

\section{Prämien und Schăden im Jahro 190r}

Deutsehe Unternehmungen

"IV 8: Die Prämien und die Schäden aus der Feuerversicherung . . . . . . . . . 246

"IV 9: Die Prämien und die Schäden aus der Sturmschäden- und der Wasserleitungsschädenversicherung . . . . . . . 250

, IV 10: Die Prämien und die Schäden ans der Einbruchdiebstahlversicherung . . 248 u. 252

\section{Ausländische Gesellschaften- Deutsches Geschäft}

" IV 11: Die Prämien und die Schäden aus der Fenerversicherung . . . . . . . . . 254

"IV 12: Die Prämien und die Schäden ans der Einbruchdiebstahlversicherung. . . . . 254

\section{Betriebsrechnung fitr 1907}

Deutsche Unternehmungen

"IV 13: Die Betriebs-Einnahmen und Ausgaben und der Betriebsgewinn der Feuerversicherung 256

IV 14: Die Betriebs-Einnahmen und Ausgaben und der Betriebsgewinn der Sturmschäden- und der Wasserleitungsschädenversicherung . 260

"IV 15: Die Betriebs-Einnahmen und Ausgaben und der Betriebsgewinn der Einbruchdiebstahlversicherang . . . . . . . . . 258 u. 262 "IV 16: Das Ergebnis der Vermögensverwaltung . 264 
Ausländische GesellschaftenGesamtges chäft

"IV 17: Die Betriebs-Einnahmen und Ausgaben aus der Feuerversicherung . . . . . . . 266

"IV 18: Die Betriebs-Einnahmen und Ausgaben aus der Einbrachdiebstahiversicherung . . . 266

\section{Bllanz fir den Sehluß des Jahres 1907}

Deutsche Unternehmungen

Tab. IV 19: Bilanz . . . . . . . . . . . . 268

"IV 20: Die Art der Kapitalanlagen und der Aktivforderungen . . . . . . . . . . . 276

„ IV 21: Die Verteilung des Gewinns . . . . . 280

" IV 22: Die Art und der Betrag der Ende 1907 vorhandenen Wertpapiere (Nennwert) . . . 284

A usländische Gesellschaften

"IV 23: Bilanz . . . . . . . . . . . . . 288

"IV 24: Die Art der Kapitalanlagen und der Aktivforderungen . . . . . . . . . 292

\section{Gruppe V. Sonstige Versioherungezweige}

Verzeichnis der Abkürzungen für die Namen der Unternehmungen . . . . . . . . 295

\section{A. Glasversicherung}

Versicherungsbestand Ende 1906 und Ende 1907 Deutsche Unternehmungen

„V 1: Zahl und Betrag der Glasversicherungen - Gesamtgeschäft. . . . . . . . . 298

,

V 2: Zahl und Betrag der Glasversicherungen

- Inländisches Geschäft . . . . . 300

\section{Ausländische Gesellschaften-} Deutsches Geschät

”

V 3: Zahl und Betrag der Glasversicherungen . 302
Prämien und Sehkden im Jahre 1907

Selto

"V 4: Die Prämien und die Schäden aus dem Glasversicherungsgeschäfte der deutschen Unternehmungen . . . . . . . . . . . 304

Tab. V 5: Die Prämien und die Schäden aus dem deutschen Glasversicherungsgeschäfte der ausländischen Gesellschaften . . . . . 302

\section{Betriebsrechnung filr 1907}

Deutsche Gesellschaften

" V 6: Die Betriebs-Einnahmen und Ausgaben and der Betriebsgewinn der Glasversicherung 306

" V 7: Das Ergebnis der Vermögensverwaltung . 312

Bilanz fur den SchluB des Jahres 1907

Deutsche Gesellschaften

" V 8: Bilanz . . . . . . . . . . . . . 308

"V 9: Die Art der Kapitalanlagen und der Aktivforderungen . . . . . . . . . . . 314

"V 10: Die Verteilung des Gewinns . . . . . 313

"V 11: Die Art und der Betrag der Ende 1907 vorhandenen Wertpapiere (Nennwert) . . . 314

B. Kantions-, Garantie-, Kredit-, Maschinen-, Banlastund Wertgegenstände-Versicherung

Deutsche Gesellschaften

„V 12: Zahl und Betrag der Versicherungen . . 316

"V 13: Die Prämien und die Schäden . . . . 320

"V 14: Die Betriebs-Einnahmen und Ausgaben und der Betriebsgewinn . . . . . . . 322

\section{Kaskoversicherung}

Deutsche Unternehmungen

"V 15: Zahl und Betrag der Kaskoversicherungen 330

"V 16: Gewinn- und Verlustrechnung . . . . 324

"V 17: Vermögensübersicht . . . . . . . . 328

Beriehtigungen . . . . . . . . 331 